\title{
The Big Five default brain: functional evidence
}

\author{
Adriana Sampaio $\cdot$ José Miguel Soares $\cdot$ \\ Joana Coutinho $\cdot$ Nuno Sousa $\cdot$ Óscar F. Gonçalves
}

Received: 6 December 2012/ Accepted: 5 July 2013/Published online: 24 July 2013

(C) Springer-Verlag Berlin Heidelberg 2013

\begin{abstract}
Recent neuroimaging studies have provided evidence that different dimensions of human personality may be associated with specific structural neuroanatomic correlates. Identifying brain correlates of a situation-independent personality structure would require evidence of a stable default mode of brain functioning. In this study, we investigated the correlates of the Big Five personality dimensions (Extraversion, Neuroticism, Openness/Intellect, Agreeableness, and Conscientiousness) and the default mode network (DMN). Forty-nine healthy adults completed the NEO-Five Factor. The results showed that the Extraversion (E) and Agreeableness (A) were positively correlated with activity in the midline core of the DMN, whereas Neuroticism $(\mathrm{N})$, Openness $(\mathrm{O})$, and Conscientiousness (C) were correlated with the parietal cortex
\end{abstract}

A. Sampaio and J. M. Soares share equal first authorship.

Electronic supplementary material The online version of this article (doi:10.1007/s00429-013-0610-y) contains supplementary material, which is available to authorized users.

A. Sampaio $(\bowtie) \cdot J$. Coutinho · Ó. F. Gonçalves

Neuropsychophysiology Lab, CIPsi, School of Psychology,

University of Minho, 4710-057 Braga, Portugal

e-mail: adriana.sampaio@psi.uminho.pt

J. M. Soares · N. Sousa

Life and Health Sciences Research Institute,

University of Minho, Braga, Portugal

J. M. Soares · N. Sousa

ICVS-3Bs PT Government Associate Laboratory,

Braga/Guimarães, Portugal

Ó. F. Gonçalves

Department of Counseling and Applied Educational Psychology,

Bouvé College of Health Sciences, Northeastern University,

Boston, USA system. Activity of the anterior cingulate cortex was positively associated with A and negatively with C. Regions of the parietal lobe were differentially associated with each personality dimension. The present study not only confirms previous functional correlates regarding the Big Five personality dimensions, but it also expands our knowledge showing the association between different personality dimensions and specific patterns of brain activation at rest.

Keywords Personality · Imaging $\cdot$ fMRI $\cdot$ Default mode network · Brain - Rest

\section{Introduction}

The question of whether the behavior is situation- or personality-specific is probably one of the longest standing debates in psychology (Mischel 1968). Several resolutions of the debate have been advanced due to the need for a refined conceptualization of personality dimensions (Fleeson and Noftle 2009), namely by building on the contributions of what is coming to be known as personality neuroscience (DeYoung 2010). In fact, personality neuroscience methods can be instrumental in identifying neurobiological correlates of different personality dimensions. What is required is a reliable model of different personality dimensions, such as The Big Five model developed by Costa and McRae (1992), as well as a methodology capable of identifying consistent biological markers underlying different personality dimensions that are situation-independent.

Therefore, the understanding of the neuroanatomical correlates of each of the Big Five components can be a valuable tool to identify more stable/biological markers of personality. Previous studies have been attempting to map the brain regions associated with Extraversion and 
Neuroticism; higher Extraversion scores were associated with thinner cortical gray matter in regions of the right inferior prefrontal cortex (PFC) and the fusiform gyrus, whereas higher Neuroticism scores were associated with a thinner cortex in the anterior regions of the left orbitofrontal cortex (Wright et al. 2006). More recently, in a study with 116 healthy volunteers, DeYoung et al. (2010) presented the first report on the association between all the Big Five personality components and brain volumetry. Overall, the authors were able to find significant neuroanatomical correlates for most of the five personality dimensions. More specifically, Extraversion was associated with increased medial orbitofrontal cortex volume; Neuroticism with reduced volume in the dorsomedial PFC and part of the left medial temporal lobe, as well as with increased volume in the mid-cingulate gyrus; Agreeableness with an increased posterior cingulate cortex (PCC) and a decreased posterior left superior temporal sulcus volumes; Conscientiousness with increased volume in the middle left frontal gyrus; and, finally, no significant associations were found for the Openness/Intellect trait. As pointed out by the authors, this study offers initial support for the neuroanatomical dimensions of the Big Five personality trait taxonomy.

Building on these previous studies, it would be important to see if these structural neuroanatomical findings would be confirmed at the functional level. Indeed, functional studies have been providing evidence regarding an association between Extraversion and greater cerebral flow at rest (rCBF), in regions such as the anterior cingulate gyrus, right insular cortex, bilateral temporal lobes, pulvinar nucleus of the thalamus, posterior parietal lobe, and left amygdala (Johnson et al. 1999). Extraversion has also been correlated with increased glucose metabolism at rest in the orbitofrontal cortex (Deckersbach et al. 2006), and right putamen (Kim et al. 2008). Neuroticism has been associated with decreased resting regional cerebral glucose metabolism in the medial PFC (Kim et al. 2008), and insular cortex (Deckersbach et al. 2006). Finally, higher Openness/Intellect scores were correlated with resting state $\mathrm{rCBF}$ in the dorsolateral $\mathrm{PFC}$, the anterior cingulate gyrus, and the orbitofrontal cortex (Sutin et al. 2009).

Overall, these studies provide initial support for the existence of associations between brain activity in several brain regions during rest (particularly the medial PFC) and Neuroticism and Extraversion. Nevertheless, this initial evidence, a full understanding of the relationship between an integrated overview of the Big Five personality dimensions and specific patterns of brain activation is still lacking. As suggested by DeYoung et al. (2010), future studies should provide an account of data that may address all brain systems simultaneously. Additionally, identifying the brain correlates of a stable personality structure would require evidence of a stable default mode of brain functioning associated with the different personality dimensions.
To explore brain functioning that is characteristic of different personality dimensions in a task-independent context, we decided to analyze the correlates of the Big Five and the default mode network (DMN) (Raichle et al. 2001), taking into account the specificity of this resting state network (RSN) in internal processing and previous evidence suggesting that individual differences in DMN activity could be able to elucidate differences in personality (Wei et al. 2012). The DMN is a network of brain cortical areas that present high metabolic activity when the brain is "at rest" and the individual is not focused on any external demand. This RSN corresponds to a high degree of functional connectivity between various interacting brain areas. Typically, the DMN comprises areas of the PCC and adjacent precuneus $(\mathrm{PCu})$; the medial prefrontal cortex (MPFC); medial, lateral and inferior parietal cortex (Raichle et al. 2001), and medial temporal cortex (Buckner et al. 2008). The DMN has been associated with multiple and dissociated components thought to serve important cognitive and emotional functions (Andrews-Hanna et al. 2010), such as supporting internal mental activity detached from the external world (Mason et al. 2007), autobiographical memory (Raichle and Snyder 2007), integrating cognitive and emotional processing (Greicius et al. 2003), and connecting internal and external attention in monitoring the world around us. Therefore, taking into account evidence showing that the DMN, a stable network of the brain, comprising multiple and dissociated components (Andrews-Hanna et al. 2010), we hypothesized that these may also be differentially related to stable personality traits.

While there is substantial evidence for an association between DMN abnormalities and psychiatric disorders [cf. (Cherkassky et al. 2006; Kennedy and Courchesne 2008; Zhou et al. 2007)], studies exploring the functional significance of DMN patterns for stable personality dimensions are still scarce. However, indirect evidence supports the idea that functional connectivity patterns may underlie the Big Five model personality organization (Wei et al. 2011; Volkow et al. 2011). For example, Volkow et al. (2011) studied the role of different brain regions' glucose metabolic activity at rest in positive emotionality (a personality trait associated with well-being, achievement/motivation and social closeness). The authors showed that this personality dimension was positively correlated with metabolism in the orbitofrontal, anterior cingulate, middle and lateral frontal, precuneus, parietal, superior and middle temporal and fusiform cortices, while negative emotionality and constraint were not correlated with any of these brain regions. As pointed out by the authors, some of these regions (the precuneus, superior parietal lobe) are components of the DMN, which indicates that increased activity of the DMN at rest may represent a neurobiological marker for a positive emotionality trait (Volkow et al. 2011). In 
addition, there is also evidence that personality traits predict resting state functional connectivity (RSFC). Adelstein et al. (2011) using a priori seeds, showed that Neuroticism and Extraversion predicted connectivity between seed regions (precuneus and ACC) and dorsomedial PFC and lateral paralimibic regions, respectively. Openness to Experience predicted RSFC with the midline 'hubs' of the DMN and dorsolateral prefrontal activity; Agreeableness predicted RSFC with posteromedial extrastriate regions and Conscientiousness predicted RSFC with the medial temporal lobe. Building on previous research demonstrating that several brain regions are associated with specific personality dimensions and that a brain default network may underlie the stability of distinct, stable psychological conditions; the objective of the current study is to explore the relationship between the personality dimensions described by the Big Five model and the overall DMN's activity patterns. Therefore, we expected that the midline core of the DMN (posterior cingulate, $\mathrm{PCu}$, and MPFC) would be differently associated with personality traits (Andrews-Hanna et al. 2010) related with self-referential processing (e.g., autobiographical conditions, self-relevant and affective decisions) as Neuroticism, Agreeableness, and Extraversion (Deckersbach et al. 2006; Kim et al. 2008; Adelstein et al. 2011; Johnson et al. 1999; Cavanna and Trimble 2006). In contrast, the parietal lobe and medial temporal cortex systems would be related with Openness to Experience/Intellect and Conscientiousness, personality traits more related with non-self processing (e.g., integration of cognitive and emotional processing, connecting internal and external attention, performing episodic judgments about the future) (DeYoung et al. 2010; Behrmann et al. 2004).

\section{Methods and materials}

\section{Participants}

Forty-nine healthy volunteers participated in this study (30 female and 19 male) with a mean age of $25.0(\mathrm{SD}=5.3$ ) years, ranging from 19 to 52 years. Participants were recruited by informal advertising of the study. The study was approved by the Human Ethics Committee of the S. Marcos Hospital, in which data were collected. After signing the written informed consent, all the participants underwent a session of personality assessment followed by an fMRI scanning session.

Personality assessment

All subjects completed the Portuguese adaption of the NEO-Five Factor Inventory (NEO-FFI) (Costa and McCrae 1995). The NEO-FFI is the short version of the NEO-PIR.
It is a psychological personality inventory that measures the five personality dimensions described by Costa and McCrae: Extraversion, Agreeableness, Conscientiousness, Neuroticism, and Openness to Experience. The instrument has 60 items (12 per domain) answered on a five-point Likert scale, ranging from "strongly disagree" to "strongly agree." NEO-FFI has good internal consistency for the different subscales $(N=0.79, E=0.79, \quad O=0.80$, $A=0.75$, and $C=0.83$ ). In addition, the test-retest reliability and the external validity of the instrument are high. The controversy about the 5-dimension solution for the debate on the number of basic personality traits is in part due to the fact that the Big Five are not totally independent dimensions. In a recent meta-analysis, van der Linden et al. (2010) collated the results of 212 Big Five studies that reported intercorrelations among Big Five measures and estimated the matrix of true intercorrelations (Cavanna and Trimble 2006), concluding that the Big Five intercorrelate. Specifically, Neuroticism tended to correlate negatively with other dimensions $(N-O: r=-0.17 ; N-C: r=-4,3$, $N-E: r=-0.36, N-A: r=-0.36)$, while the other personality dimensions tended to correlate positively (ex: $E-O: r=0.43, C-A: r=0.43, C-E: r=0.29, A-O$ : $r=0.21$ and $C-O: r=0.20)$. Using confirmatory factor analyses they found also that the model in which the Big Five was assumed to be uncorrelated (orthogonal) did not fit the data. This intercorrelation nature of personality measures was also considered in our study $(N-E$ : $r=$ $-0.28, \quad p=0.05 ; A-O: \quad r=0.49, \quad p=0.000, A-C$ : $r=0.41, p=0.003$ ), when performing the correlations with the DMN activity. For this study, all NEO-FFI variables followed a normal distribution, assessed by Kolmogorov-Smirnov and Shapiro tests [Neuroticism: $\mathrm{K}-\mathrm{S}(49)=0.08, p=0.20$; Extraversion: $\mathrm{K}-\mathrm{S}(49)=0.07$, $p=0.20$; Openness/Intellect: $\mathrm{K}-\mathrm{S}(49)=0.11, p=0.18$, Agreeableness: $\mathrm{K}-\mathrm{S}(49)=0.10, \quad p=0.20$, Conscientiousness: $\left.\mathrm{S}-\mathrm{W}^{1}(49)=0.98, \quad p=0.05\right]$. We did not observe an association between sex and personality dimensions (Chi square test, $p>0.05$ ) or between age and personality scores $(p>0.05)$. Our participants' scores in the different subscales, along with demographic data, are summarized in Table 1.

\section{fMRI acquisition}

The brain's gradient-echo echo-planar imaging (EPI) BOLD fMRI acquisition sequence was conducted on a clinically approved Siemens Magnetom Avanto $1.5 \mathrm{~T}$ in the S. Marcos Hospital. During the task-free acquisition,

\footnotetext{
1 All variables but Conscientiousness followed a normal distribution sample with $\mathrm{p}<0.05$ in both tests $(\mathrm{K}-\mathrm{S}$ and $\mathrm{S}-\mathrm{W})-$ here we included only S-W test.
} 
Table 1 Main demographic and personality measures

\begin{tabular}{ll}
\hline Main demographic and personality measures \\
\hline Number of subjects & 49 \\
Men/women & $19 / 30$ \\
Mean age, SD (range) & $M=25.0, \mathrm{SD}=5.3$ \\
NEO-FFI scores & \\
Neuroticism & $M=22.5, \mathrm{SD}=4.71$ \\
Extraversion & $M=30.5, \mathrm{SD}=4.94$ \\
Openness/Intellect & $M=29.1, \mathrm{SD}=5.32$ \\
Agreeableness & $M=28.49, \mathrm{SD}=7.09$ \\
Conscientiousness & $M=32.28, \mathrm{SD}=5.44$ \\
\hline
\end{tabular}

subjects were instructed to keep the eyes closed and to think about nothing particular. This axial whole brain sequence with 100 volumes was obtained with $\mathrm{TR}=3 \mathrm{~s}$, $\mathrm{TE}=50 \mathrm{~ms}, \quad \mathrm{FA}=90^{\circ}, \quad$ in-plane $\quad$ resolution $=$ $3.4 \times 3.4 \mathrm{~mm}^{2}, 30$ interleaved slices, thickness $=5 \mathrm{~mm}$, imaging matrix $64 \times 64$, and $\mathrm{FoV}=220 \mathrm{~mm}$.

\section{Image processing}

Prior to further processing and analysis of the different image sequences, all the images were inspected to confirm that they were not affected by critical head motion and participants had no brain lesions. To achieve signal stabilization and allow subjects to adjust to the scanner noise, the first five volumes (15 s) were discarded. Data were further pre-processed using SPM8 (Statistical Parametrical Mapping, version 8, http://www.fil.ion.ucl.ac.uk). Functional MRI data were corrected for errors in slice timing, using first slice as reference and SPM8's Fourier phase shift interpolation, to reduce different slice time acquisition. Images were realigned to the mean image to correct head motion with a six-parameter rigid-body spatial transformation and estimation was performed at 0.9 quality, 4-mm separation, 5-mm FWHM smoothing kernel using second degree B-Spline interpolation, and spatially normalized to the Montreal Neurological Institute (MNI) standard coordinate system using SPM8 EPI template and trilinear interpolation. Data were then resampled every $3 \mathrm{~mm}$ using sinc interpolation, smoothed to decrease spatial noise with a $6 \mathrm{~mm}$, full-width, half-maximum, Gaussian kernel and temporally band-pass filtered $(0.01-0.08 \mathrm{~Hz})$ to reduce physiological noise. All subjects displayed head motion less than $2 \mathrm{~mm}$ in translation or $2^{\circ}$ in rotation.

Independent component analysis

To study the functional networks involved in the task-free BOLD sequence, spatial ICA analysis was performed using the Group ICA 2.0d of fMRI Toolbox (GIFT, http://www. icatb.sourceforge.net) (Calhoun et al. 2001a; Correa et al. 2005). The ICA analysis consists in extracting the individual spatial independent maps and their related time courses. The reduction of dimensionality of the functional data and computational load was performed with principal component analysis (PCA). The number of independent components estimated was 20 for each subject, based a good trade-off (clustering/splitting) between preserving the information in the data while reducing its size (Beckmann et al. 2005; Calhoun et al. 2001b). ICA calculation was then performed using the iterative Infomax algorithm. The ICASSO tool was used to control the ICA reliability. Twenty computational runs were made on the dataset, during which the components were being recomputed and compared across runs and the robustness of the results was ensured (Wei et al. 2012). The independent components were obtained and each voxel of the spatial map was expressed as a $t$ statistic map, which was finally converted to a $z$ statistic that characterizes the degree of correlation of the voxel signal with the component time course, providing indirectly a degree of functional connectivity within the network (Beckmann et al. 2005; Kunisato et al. 2011). The components were sorted and spatially correlated with the default mode template from GIFT for DMN identification and were also visually inspected. Finally, the best-fit component of each individual ( $z$ maps) was used to perform group statistical analyses (second level analyses).

\section{Statistical analyses}

For the group study, the SPM8's level General Linear Model (GLM) method was used to analyze the pre-processed datasets. All the individual default mode $z$ maps were included in the same group and a one-sample $t$ test $(p<0.05$ FWE corrected for multiple comparisons, extent threshold $k=10$ voxels) was used to study the global pattern of DMN activation. Then a multiple regression (with positive and negative correlations) was performed, using all subjects in the study and each one of the Big Five model personality dimensions, controlling for the other four dimensions and for age and gender. Results were considered significant at a corrected for multiple comparisons $p<0.05$ threshold (combined height threshold $p<0.01$ and a minimum cluster size $=24$, using $\mathrm{FWHM}=8 \mathrm{~mm}, \mathrm{rmm}=5$ and 1,000 iterations), determined by Monte Carlo simulation program (AlphaSim). The resulting statistical maps ( $t$ and $z$ statistics) represent the strength of the association between personality dimensions and DMN functional activation. These were presented using the same DMN template mask to sort the components that were applied to the whole brain activation patterns. Finally, $T$ scores for the DMN areas were reported and converted into a goodness of fit score $\left(r^{2}\right)$ and $95 \%$ CI. 

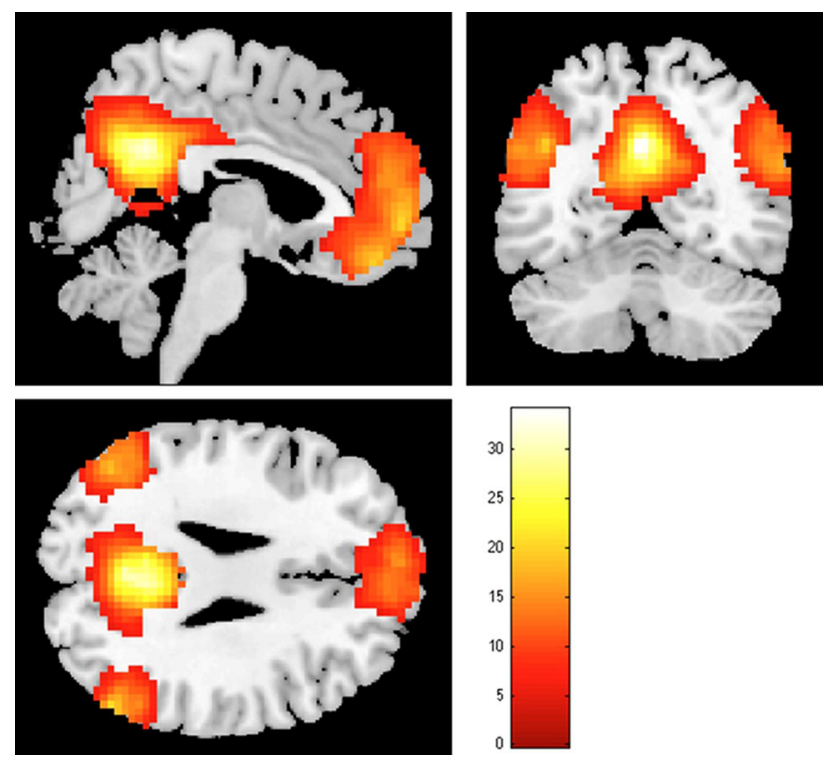

Fig. 1 Group activation pattern of DMN ( $p<0.05$ FWE corrected)

Within the template, only the typical DMN regions were reported and anatomical labeling was assigned by a combination of visual inspection and Anatomical Automatic Labeling atlas (AAL) (Krebs et al. 2009).

\section{Results}

\section{DMN results}

The DMN was identified in the resting state conditions at the group level, and four main components were observed, namely the PCCs and $\mathrm{PCu}$, the MPFC, the bilateral inferior parietal cortex (IPC), and the left inferior temporal cortex (ITC) (Adelstein et al. 2011; see Fig. 1). The statistics of the group DMN pattern are represented in Table 2. The statistics of the group DMN pattern are represented in Table 2.

\section{Neuroticism and DMN components}

Within the DMN, increased Neuroticism scores were associated with decreased activity of the right superior parietal cortex $(x=30, y=-72, z=54, \quad T=3.02$, $r^{2}=0.18$, IC $0.05-0.23$ ) (see supplemental table and Figs. 2, 3).

\section{Extraversion and DMN components}

Significant positive correlations were found between Extraversion and the DMN, namely, the right precuneus $\left(x=18, \quad y=-51, \quad z=15 ; \quad T=3.74, \quad r^{2}=0.25, \quad\right.$ IC 0.04-0.11), bilateral superior parietal lobe (right: $x=15$,
Table 2 Group statistics-DMN activation (FWE $<0.05$ corrected, extent threshold $k=10$ voxels)

\begin{tabular}{llll}
\hline Regions & $Z$ & $k$ & MNI coordinates $(x, y, z)$ \\
\hline Precuneus & $>8$ & 2,134 & $0,-60,33$ \\
Left parietal lobe & $>8$ & 878 & $-42,-63,33$ \\
Right parietal lobe & $>8$ & 676 & $54,-66,30$ \\
Medial prefrontal & $>8$ & 1,835 & $3,57,-3$ \\
Left medial temporal & 6.37 & 18 & $-57,-42.0$ \\
\hline
\end{tabular}

$y=-66, z=66, T=3.72, r^{2}=0.25$, IC 0.05-0.18; left: $x=-39, \quad y=-51, \quad z=63, \quad T=3.49, \quad r^{2}=0.23, \quad$ IC $0.04-0.15)$ and left inferior parietal lobe $(x=-42, \mathrm{y}=$ $-60, z=54, T=3.21, \quad r^{2}=0.20$, IC $0.05-0.21 \quad$ (see supplemental table and Figs. 2, 4).

\section{Openness/Intellect and DMN component}

Increased Openness/Intellect was associated with increased activity in the right inferior parietal lobe $(x=48, y=$ $-57, z=27, T=5.02, r^{2}=0.38$, IC 0.12-0.28) and with decreased activity in bilateral superior parietal cortex (right: $x=42, y=-45, z=66 T=4.13, r^{2}=0.29$, IC 0.07-0.21; left: $x=-24, y=-72, z=54, \quad T=3.36$ $r^{2}=0.22$, IC 0.06-0.211) and in the left precuneus $(x=$ $-15, \quad y=-66, \quad z=57, \quad T=3.91, \quad r^{2}=0.27, \quad$ IC 0.04-0.12) (see supplemental table and Figs. 2, 5).

\section{Agreeableness and DMN components}

The BOLD activity in specific components of the DMN [the MPFC and ACC $(x=9, y=42, z=0, T=4.31$ $r^{2}=0.31, \quad$ IC $0.03-0.08 ; x=-6, y=39, \quad z=-6$, $T=3.61, r^{2}=0.24$, IC $\left.0.03-0.10\right]$ was positively correlated with Agreeableness. Negative associations between DMN and Agreeableness were evident in the right superior parietal lobe $(x=30, y=-72, z=60, \quad T=2.81$, $r^{2}=0.16$, IC 0.03-0.16) (see supplemental table and Figs. 2, 6).

\section{Conscientiousness and DMN components}

Finally, increased Conscientiousness scores were positively associated with right superior parietal cortex $(x=33$, $y=-78, z=51, T=3.36, r^{2}=0.22$, IC $\left.0.04-0.16\right)$ and negatively associated with brain activity in bilateral precuneus $\left(x=-3, y=-78, z=54, T=4.75, r^{2}=0.36\right.$, IC $0.13-0.39 ; x=18, y=-72, \quad z=45, \quad T=3.49$, $r^{2}=0.23$, IC $\left.0.04-0.13\right)$ and bilateral ACC $(x=-3$, $y=48, z=15, T=3.70, r^{2}=0.25$, IC 0.04-0.12; $x=6$, $y=36, z=9, T=3.57, r^{2}=0.24$, IC $0.03-0.87$ ) (see supplemental table and Figs. 2, 7). 
(a)

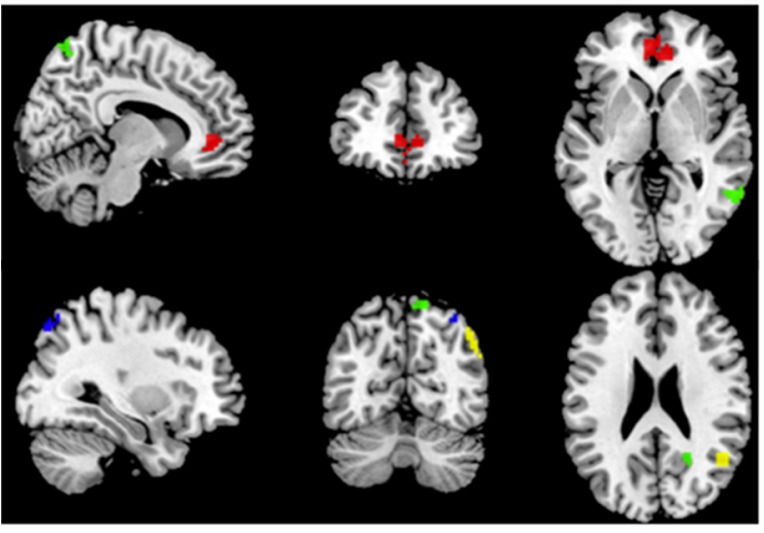

Agreeableness Consciousness (b)

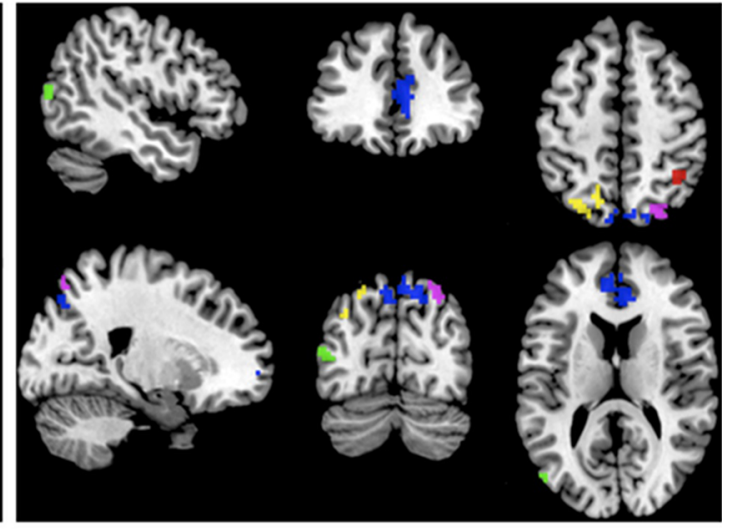

Extraversion Neuroticism
Fig. 2 a Positive correlations $(p<0.05$ corrected for multiple comparisons) between Neuroticism (pink), Extraversion (green), Openness/Intellect (yellow), Agreeableness (red), Conscientiousness (blue) scores and DMN components; b negative correlations
( $p<0.05$ corrected for multiple comparisons) between Neuroticism (pink), Extraversion (green), Openness/Intellect (yellow), Agreeableness (red), Conscientiousness (blue) scores and DMN components

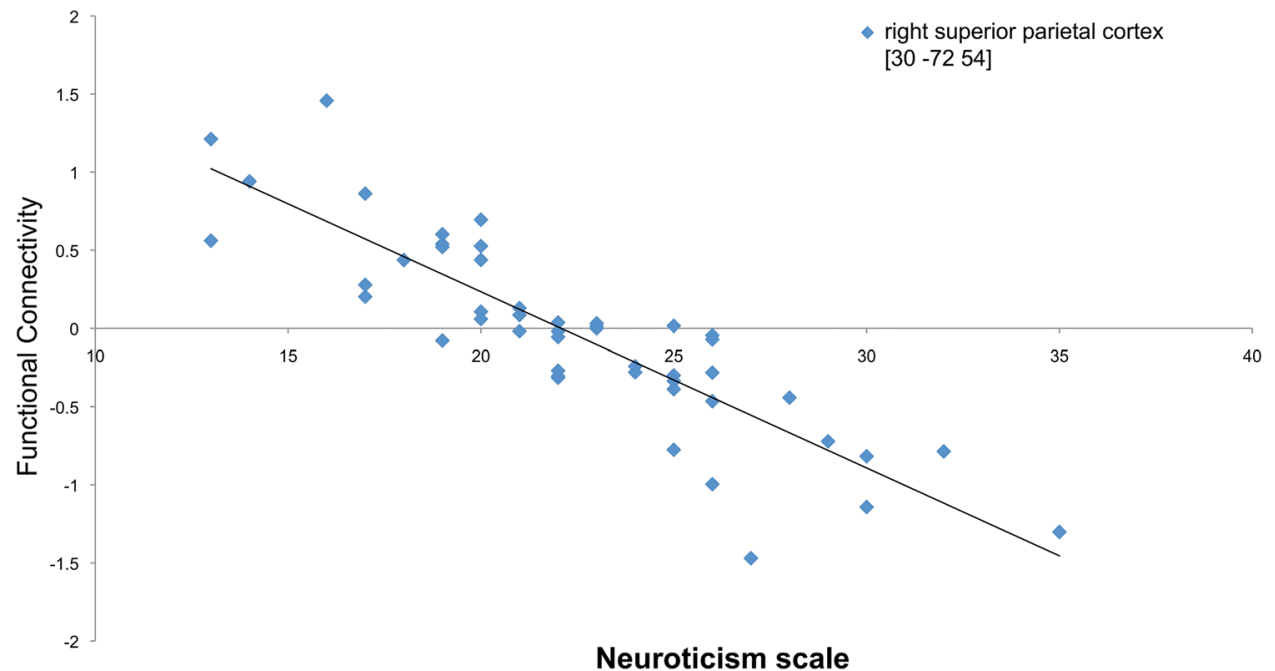

Fig. 3 Correlation plot for DMN components (functional connectivity) and Neuroticism scale

\section{Discussion}

To the best of our knowledge, this is one of the first studies exploring the association between personality measures and overall DMN functional activity. There is already an evidence showing a relationship between the five factor personality traits and RSFC between seed regions positioned within the anterior cingulate and the precuneus (cognitive and affective 'hubs') and functional connectivity with several brain areas within and outside the DMN (Adelstein et al. 2011). In this study, we performed a more restricted analysis, circumscribed to only typical DMN regions.

Overall, we found evidence that the Extraversion (E), and Agreeableness (A) dimensions were positively correlated with the activity in the midline core of the DMN, whereas Conscientiousness (C) and Openness (O) scores were positively correlated with activation in the parietal cortex systems. This is in line with our predictions, namely that the midline core of the DMN would be more associated with personality traits characterized by selfreferential processing ( $\mathrm{E}$ and $\mathrm{A}$ ), while the parietal lobe system would be more related with non-self processing traits $(\mathrm{C}$ and $\mathrm{O})$.

In fact, Agreeableness was the only personality trait positively associated with the MPFC and ACC, a DMN component associated with social awareness, including the ability to attribute mental state to others (Gusnard et al. 2001; Lane et al. 1998). Indeed, stronger activity in the midline core of the DMN has been related with preferential self-related activity (Andrews-Hanna et al. 2010) as emotional state attribution, personal significance, motivation to 


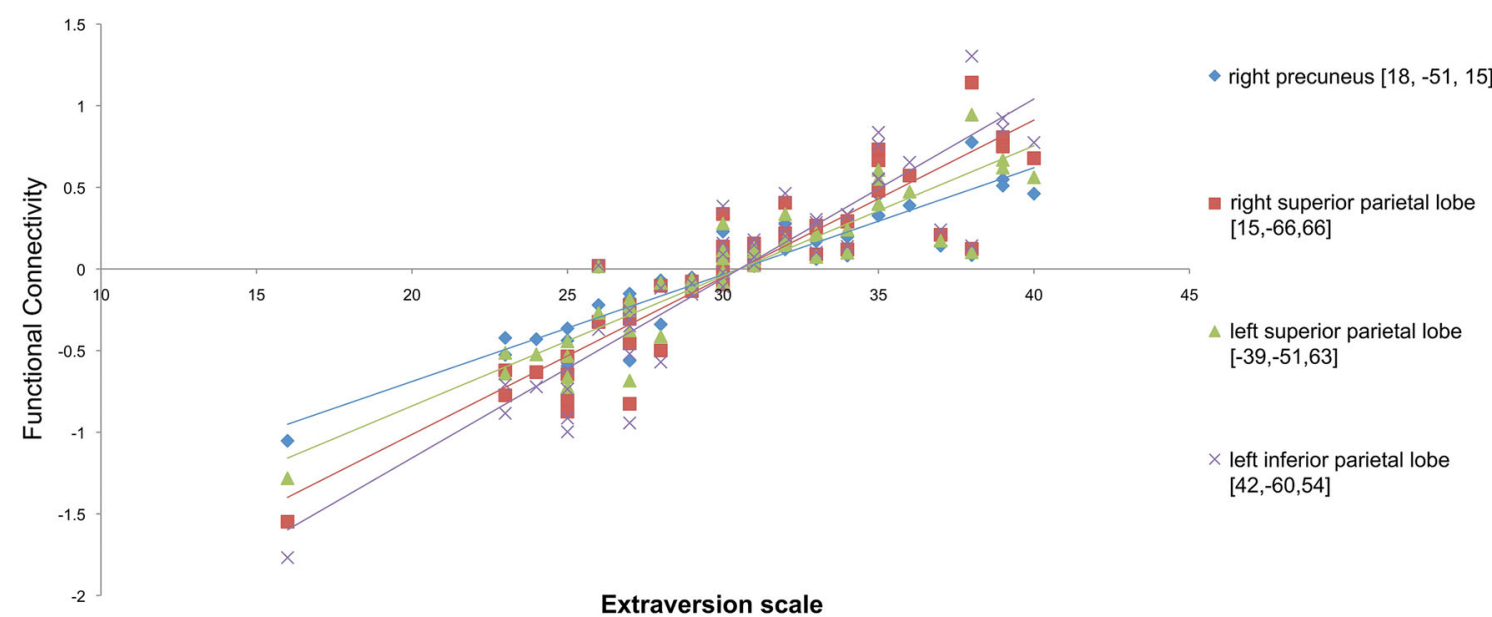

Fig. 4 Correlation plot for DMN components (functional connectivity) and Extraversion scale

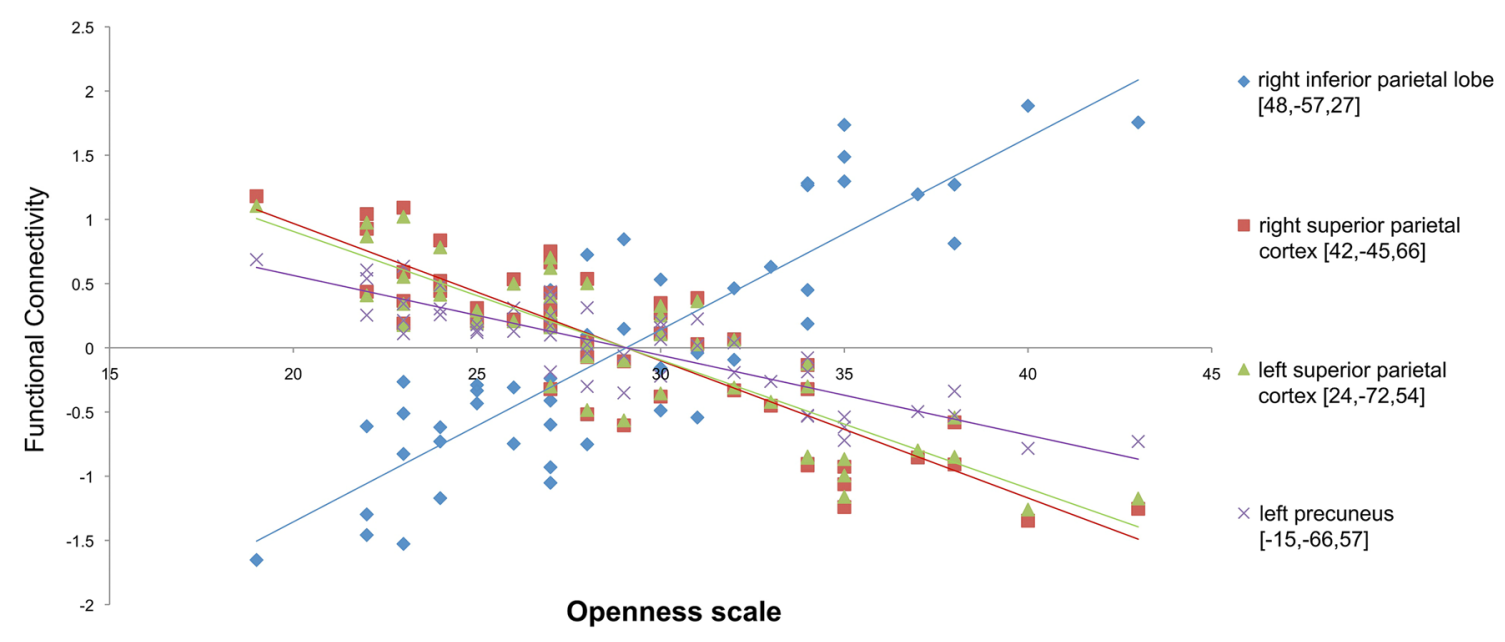

Fig. 5 Correlation plot for DMN components (functional connectivity) and Openness scale

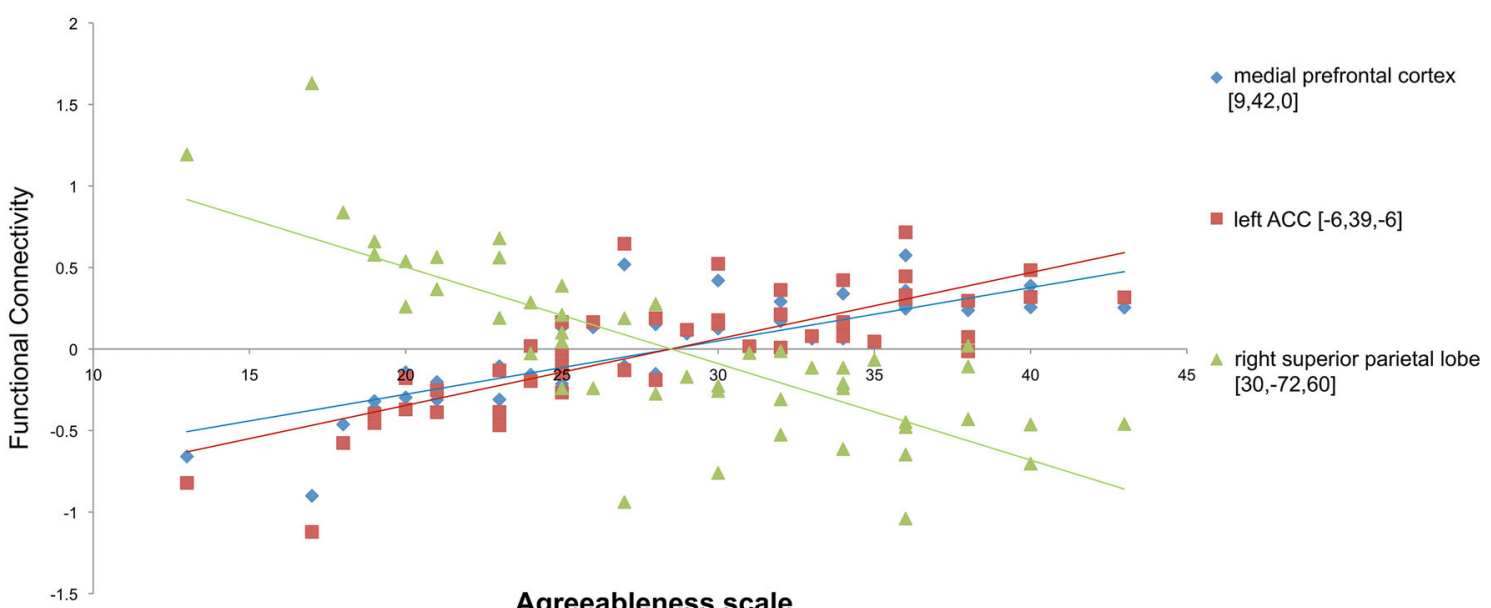

Agreeableness scale

Fig. 6 Correlation plot for DMN components (functional connectivity) and Agreeableness scale 


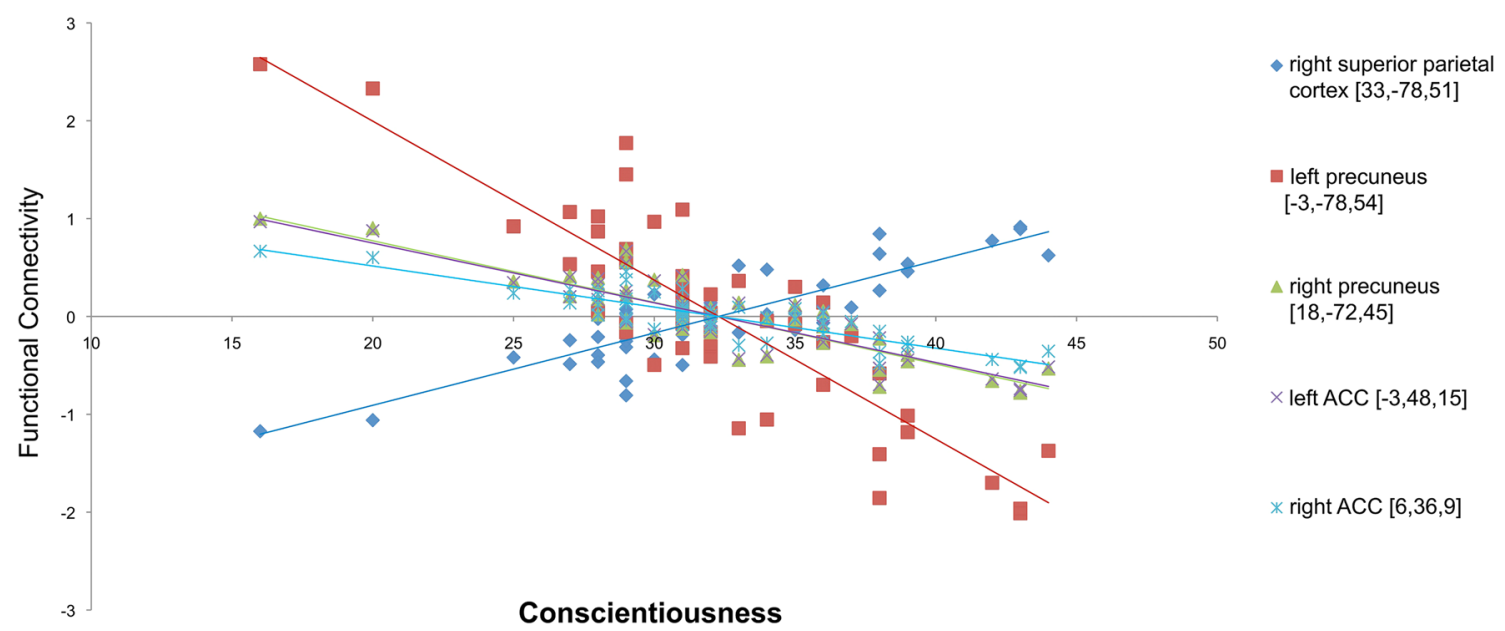

Fig. 7 Correlation plot for DMN components (functional connectivity) and Conscientiousness scale

positive reinforcement, and social cognition. Together, these dimensions reflect a pro-social orientation and the ability to respond to the needs of others in an empathic way, all of which are social-cognitive tasks (Extraversion and Agreeableness-related dimensions) subserved by the midline core of the DMN (Andrews-Hanna et al. 2010). Additionally, and consistent with other studies, Extraversion was also found to be positively associated with the precuneus (Kunisato et al. 2011; Wei et al. 2012; Ryman et al. 2011), a brain region playing an important role in emotional regulation and self-related mental representations (Cavanna and Trimble 2006).

In accordance with this hypothesis, we observed that these midline core areas (ACC and precuneus) were negatively associated with a personality dimension characterized by less engagement in social-oriented tasks and selfprocessing, but more self-regulation processes, such as mental schemes elaboration, inhibition, self-discipline, and planning (Conscientiousness). In comparison with the midline core of the DMN, the parietal cortex system displays an important functional role in attentional control, response inhibition (Garavan et al. 1999) and task switching (Sohn et al. 2000). Specifically, we observed a relationship between higher Conscientiousness and increased bilateral parietal cortex activation, confirming our prediction suggesting that parietal lobe system would be more associated with non-self processing traits. In fact, these functional properties of the parietal system have been previously associated with the Conscientiousness and Openness traits (DeYoung et al. 2010; Behrmann et al. 2004). In addition, higher Openness scores were associated with higher activation in the inferior parietal lobe, although this personality measure was negatively associated with activity in the bilateral superior parietal cortex and left precuneus. These results suggest that activity within the parietal cortex is differentially associated with the ability to process social, emotional, and sensorial stimuli. This observation is in accordance with evidence showing that parietal regions have an additional role in processing more outward-directed social or contextual stimuli (Johnson et al. 2006; Volkow et al. 2011; Johnson et al. 1999) and with data derived from our study, namely the differential relation between these brain regions and Extraversion and Neuroticism. Therefore, while Extraversion was associated with greater regional cerebral flow in the parietal cortex system (superior and inferior parietal cortex), stronger activity in the right superior parietal cortex was associated with lower Agreeableness and Neuroticism scores. Right superior parietal cortex has been associated with response to negative pictures and meaning (e.g., categorization of sentences) (Chan et al. 2008), suggesting that personality (E, A, and N) modulates differently the effects of emotional arousal and valence on brain activation (Kehoe et al. 2011), as already noted. Indeed, these results are consistent with indirect evidence showing an abnormal activity of this brain region in individuals with autism spectrum disorders when are engaged in social-oriented tasks (Greene et al. 2011).

As previously mentioned, the main objective of this study was to ascertain whether the reports on the association between structural neuroanatomy and the Big Five personality trait taxonomy would be confirmed at the functional level. As DeYoung et al. (2010) argued, although it is reasonable to think "volume tends to covary positively with function," (p.822) we must be cautious when making predictions about the direction of the effect. The present study not only confirms previous data on the functional correlates of the Big Five personality dimensions, but also expands our understanding of this relationship by showing that different personality dimensions are associated with specific patterns of activation in a brain default mode. Moreover, we did not replicate the association between Big Five traits and specific brain 
regions reported by previous studies (Adelstein et al. 2011; Sutin et al. 2009; DeYoung et al. 2010). These may be due to the different methodological approaches; specifically, the use of different resting state networks and different analysis procedures-for example, Adelstein et al. (2011) used a ROI seed based regions analysis, whereas we used an independent component analysis approach. Indeed, as the authors acknowledge, the complexity of brain-behavior interaction is such that it requires us to look for personality networks that may link different brain regions. Our findings constitute an important step in this direction, namely by identifying associations between specific personality traits and key DMN regions. Moreover, other studies have been linking personality traits with other RSNs that were not tackled in this study and should be addressed in future studies. Once data on resting activity underlying personality are more consistent, we will be able to derive important clinical implications, namely to predict which alterations in the resting state activity are likely to increase the vulnerability for several Axis I psychiatric disorders, known to be associated with specific personality traits.

Although the promising evidence found in our study, our results should be interpreted with caution due to several methodological limitations. First it is important to highlight that we used a very specific index of DMN activity (ICA $z$ scores) and our results should be interpreted taking into account that methodological option. Another limitation is the correlational nature of our data, preventing the establishment of a causal relationship between personality dimensions and DMN patterns. We believe this relationship is a complex one because the psychological tasks supported by the DMN tend to activate multiple regions within the network. Moreover, as Buckner et al. (2008) suggested, these tasks share core processes in common but differ in terms of the content and goal to which these processes are applied, which in turn may determine the transient interactions between the DMN components and other brain systems. Different personality dimensions influence the individual's tendency to engage in different cognitive or emotional functions, but the specific nature of their internal mental activity may also play a role. Indeed, this is difficult to overcome in this study since we are using resting state scans with less control over what cognitive/emotional processes are being engaged during the scan. Therefore, future studies should try to conciliate the assessment of the subjects' personality dimensions with the specific content of their spontaneous cognitive activity at rest.

Acknowledgments This research was funded by PIC/IC/83290/ 2007, which is supported by FEDER (POFC-COMPETE) and FCT. The authors acknowledge Jaime Rocha for his discussions on neuroimaging.

\section{References}

Adelstein JS, Shehzad Z, Mennes M, Deyoung CG, Zuo XN, Kelly C, Margulies DS, Bloomfield A, Gray JR, Castellanos FX, Milham MP (2011) Personality is reflected in the brain's intrinsic functional architecture. PLoS One 6(11):e27633. doi:10.1371/ journal.pone.0027633

Andrews-Hanna JR, Reidler JS, Sepulcre J, Poulin R, Buckner RL (2010) Functional-anatomic fractionation of the brain's default network. Neuron 65(4):550-562. doi:10.1016/j.neuron.2010.02. 005

Beckmann CF, DeLuca M, Devlin JT, Smith SM (2005) Investigations into resting-state connectivity using independent component analysis. Philos Trans $\mathrm{R}$ Soc Lond B Biol Sci 360(1457):1001-1013. doi:10.1098/rstb.2005.1634

Behrmann M, Geng JJ, Shomstein S (2004) Parietal cortex and attention. Curr Opin Neurobiol 14(2):212-217. doi:10.1016/j. conb.2004.03.012

Buckner RL, Andrews-Hanna JR, Schacter DL (2008) The brain's default network: anatomy, function, and relevance to disease. Ann N Y Acad Sci 1124:1-38

Calhoun VD, Adali T, Pearlson GD, Pekar JJ (2001a) A method for making group inferences from functional MRI data using independent component analysis. Hum Brain Mapp 14:140-151

Calhoun VD, Adali T, Pearlson GD, Pekar JJ (2001b) Spatial and temporal independent component analysis of functional MRI data containing a pair of task-related waveforms. Hum Brain Mapp 13:43-53

Cavanna AE, Trimble MR (2006) The precuneus: a review of its functional anatomy and behavioural correlates. Brain: J Neurol 129(Pt 3):564-583. doi:10.1093/brain/awl004

Chan SW, Harmer CJ, Goodwin GM, Norbury R (2008) Risk for depression is associated with neural biases in emotional categorisation. Neuropsychologia 46(12):2896-2903. doi:10. 1016/j.neuropsychologia.2008.05.030

Cherkassky VL, Kana RK, Keller TA, Just MA (2006) Functional connectivity in baseline resting-state network in autism. Neuroreport 17:1687-1690

Correa N, Adali T, Li Y, Calhoun V (2005) Comparison of blind source separation algorithms for FMRI using a new Matlab toolbox: GIFT. Proc IEEE Int Conf Acoust Speech Signal Process 5:401-404

Costa PT, McCrae RR (1992) Revised NEO personality inventory (NEOPI- R) and the NEO five-factor inventory (NEO-FFI) professional manual. Psychological Assessment Resources, Odessa, FL

Costa PT, McCrae RR (1995) Domains and facets: hierarchical personality assessment using the revised NEO personality inventory. J Pers Assess 64:21-50

Deckersbach T, Miller KK, Klibanski A, Fischman A, Dougherty DD, Blais MA, Herzog DB, Rauch SL (2006) Regional cerebral brain metabolism correlates of neuroticism and extraversion. Depress Anxiety 23(3):133-138. doi:10.1002/da.20152

DeYoung CG (2010) Personality neuroscience and the biology of traits. Soc Pers Psychol Compass 4(12):1165-1180

DeYoung CG, Hirsh JB, Shane MS, Papademetris X, Rajeevan N, Gray JR (2010) Testing predictions from personality neuroscience. Psychol Sci 21(6):820

Fleeson W, Noftle EE (2009) In favor of the synthetic resolution to the person-situation debate. J Res Pers 43(2):150-154

Garavan H, Ross TJ, Stein EA (1999) Right hemispheric dominance of inhibitory control: an event-related functional MRI study. Proc Natl Acad Sci USA 96(14):8301-8306

Greene DJ, Colich N, Iacoboni M, Zaidel E, Bookheimer SY, Dapretto M (2011) Atypical neural networks for social orienting 
in autism spectrum disorders. Neuroimage 56(1):354-362. doi:10.1016/j.neuroimage.2011.02.031

Greicius MD, Krasnow B, Reiss AL, Menon V (2003) Functional connectivity in the resting brain: a network analysis of the default mode hypothesis. Proc Natl Acad Sci USA 100(1): 253-258. doi:10.1073/pnas.0135058100

Gusnard DA, Akbudak E, Shulman GL, Raichle ME (2001) Medial prefrontal cortex and self-referential mental activity: relation to a default mode of brain function. In: Proceedings of the National Academy of Sciences of the United States of America

Johnson DL, Wiebe JS, Gold SM, Andreasen NC, Hichwa RD, Watkins GL, Boles Ponto LL (1999) Cerebral blood flow and personality: a positron emission tomography study. Am J Psychiatry 156(2):252-257

Johnson MK, Raye CL, Mitchell KJ, Touryan SR, Greene EJ, NolenHoeksema S (2006) Dissociating medial frontal and posterior cingulate activity during self-reflection. Soc Cogn Affect Neurosci 1(1):56-64. doi:10.1093/scan/ns1004

Kehoe EG, Toomey JM, Balsters JH, Bokde AL (2011) Personality modulates the effects of emotional arousal and valence on brain activation. Soc Cogn Affect Neurosci. doi:10.1093/scan/nsr059

Kennedy DP, Courchesne E (2008) The intrinsic functional organisation of the brain is altered in autism. Neuroimage 39:18771885

Kim SH, Hwang JH, Park HS, Kim SE (2008) Resting brain metabolic correlates of neuroticism and extraversion in young men. Neuroreport 19(8):883-886. doi:10.1097/WNR.0b013e 328300080f

Krebs RM, Schott BH, Duzel E (2009) Personality traits are differentially associated with patterns of reward and novelty processing in the human substantia nigra/ventral tegmental area. Biol Psychiatry 65(2):103-110. doi:10.1016/j.biopsych.2008.08. 019

Kunisato Y, Okamoto Y, Okada G, Aoyama S, Nishiyama Y, Onoda K, Yamawaki S (2011) Personality traits and the amplitude of spontaneous low-frequency oscillations during resting state. Neurosci Lett 492(2):109-113. doi:10.1016/j.neulet.2011.01.067

Lane RD, Reiman EM, Axelrod B, Yun LS, Holmes A, Schwartz GE (1998) Neural correlates of levels of emotional awareness. Evidence of an interaction between emotion and attention in the anterior cingulate cortex. J Cogn Neurosci 10(4):525-535

Mason MF, Norton MI, Van Horn JD, Wegner DM, Grafton ST, Macrae CN (2007) Wandering minds: the default network and stimulus independent thought. Science 315:393-395
Mischel W (1968) Personality and assessment. John Wiley, New York

Raichle ME, Snyder AZ (2007) A default mode of brain function: a brief history of an evolving idea. Neuroimage 37(4):1083-1090. doi:10.1016/j.neuroimage.2007.02.041 (discussion 1097-1089)

Raichle ME, MacLeod AM, Snyder AZ, Powers WJ, Gusnard DA, Shulman GL (2001) A default mode of brain function. Proc Natl Acad Sci 98:676-682

Ryman SG, Gasparovic C, Bedrick EJ, Flores RA, Marshall AN, Jung RE (2011) Brain biochemistry and personality: a magnetic resonance spectroscopy study. PLoS One 6(11):e26758. doi:10. 1371/journal.pone. 0026758

Sohn MH, Ursu S, Anderson JR, Stenger VA, Carter CS (2000) The role of prefrontal cortex and posterior parietal cortex in task switching. Proc Natl Acad Sci USA 97(24):13448-13453. doi:10.1073/pnas.240460497

Sutin AR, Beason-Held LL, Resnick SM, Costa PT (2009) Sex differences in resting-state neural correlates of openness to experience among older adults. Cereb Cortex 19(12):2797-2802. doi:bhp06610.1093/cercor/bhp066

van der Linden D, Nijenhuis J, Bakker AB (2010) The general factor of personality: a meta-analysis of big five intercorrelations and a criterion-related validity study. J Res Personal 44(3):315-327

Volkow ND, Tomasi D, Wang GJ, Fowler JS, Telang F, Goldstein RZ, Alia-Klein N, Woicik P, Wong C, Logan J, Millard J, Alexoff D (2011) Positive emotionality is associated with baseline metabolism in orbitofrontal cortex and in regions of the default network. Mol Psychiatry. doi:10.1038/mp.2011.30

Wei L, Duan X, Yang Y, Liao W, Gao Q, Ding JR, Zhang Z, Zeng W, Li Y, Lu G, Chen H (2011) The synchronization of spontaneous BOLD activity predicts extraversion and neuroticism. Brain Res 1419:68-75. doi:10.1016/j.brainres.2011.08.060

Wei L, Duan X, Zheng C, Wang S, Gao Q, Zhang Z, Lu G, Chen H (2012) Specific frequency bands of amplitude low-frequency oscillation encodes personality. Hum Brain Mapp. doi:10.1002/ hbm. 22176

Wright CI, Williams D, Feczko E, Barrett LF, Dickerson BC, Schwartz CE, Wedig MM (2006) Neuroanatomical correlates of extraversion and neuroticism. Cereb Cortex 16(12):1809-1819. doi:bhj11810.1093/cercor/bhj118

Zhou Y, Liang M, Tian L, Wang K, Hao Y, Liu H, Liu Z, Jiang T (2007) Functional disintegration in paranoid schizophrenia using resting-state fMRI. Schizophr Res 97:194-205 